Die nachfolgende "Erklärung zur gegenwärtıgen Einstellungspraxıs beim Offentlichen Dienst im Zusammenhang mit den sogenannten Radikalenerlassen vom 22. Januar 1975 haben 100 Professoren der baden-württembergischen Universtäten Konstanz, Freiburg, Heldelberg, Tübingen und Stuttgart unterzeichnet.

Die gegenwärtige Praxis bei der Einstellung in den Offentlichen Dienst soll nach den ihr zugrundeliegenden Erklärungen die verfassungskonforme Bewältıgung der Aufgaben des Offentlichen Dienstes sichern. Durch die an die sogenannten Radikalenerlasse anknüpfende Ausforschungs- und Beurteilungspraxis sowie durch eine Reihe bekannt gewordener Ablehnungsfälle ist jedoch fraglich geworden, ob die Landesregierungen (insbesondere diejenige Baden-Württembergs) die Freiheiten des Art. S Grundgesetz und die yorgeschriebene Treue zur Verfassung noch in einem abgewogenen Verhältnis zueinander halten. Gegenüber der eingetretenen bedrohlichen Verunsıcherung sei hier das für ein vernünftig verstandenes demokratisches Gemennwesen Selbstverständliche in Erinnerung gerufen:

I. Das Grundgesetz stellt es gemäß Art. Is frei, Grund und Boden, Naturschätze und Produktionsmittel zum Zwecke der Vergesellschaftung durch ein Gesetz, das Art und Ausmaß der Entschädigung regelt, im Gemeineigentum oder in anderer Form der Gemeinwirtschaft zu überführen. Es enthält in diesem Sinne keine Festlegung auf die marktwirtschaftliche oder kapitalistische Wirtschaftsordnung. Es gebietet überdies gemäß Art. I 4, die Eigentumsordnung der Republik daran zu messen, ob sie dem „Wohle der Allgemeinheit" dient. Daraus folgt zunächst, daß Meınungen und wissenschaftliche Theorien, die der Kritik der marktwirtschaftlichen Ordnung verpflichtet sind, gegebenenfalls auch das System dieser Ordnung grundlegend verändernde Alternativen vortragen, dem Auftrag des Grundgesetzes aktıv entsprechen. Auch daß hier wie überall Irren menschlich ist, kann das entsprechende Bemühen nicht als verfassungsfeindlich diskreditieren.

2. Es gilt darüber hinaus, daß für grundlegende Änderungen des Systems der marktwirtschaftlichen Ordnung gemäß Art. I 4 und is des Grundgesetzes aktiv politisch eingetreten und in Ubereinstimmung mit Gesetz und Verfassung gehandelt werden darf. Die Einstellungspraxis im Offentlichen Dienst darf daher, soweit es sich nicht um sogenannte politische Beamte handelt, einer solchen Fortentwicklung der Verfassung nicht entgegentreten. Sie würde sonst den Angehörigen des Offentlichen Dienstes einen bestimmten (unter den durch Verfassung und Gesetz zugelassenen) Willen vorschreiben und damit das Recht der freien Berufswahl (Art. I 2 Abs. I Grundgesetz) oder das Recht, seine Meinung in Wort, Schrift und Bild frei zu äußern und zu verbreiten (Art. s, Abs. I Grundgesetz) verfassungswidrig, entsprechend dem in totalitären Systemen Üblichen, einschränken. Für den Bereich wissenschaftlicher Forschung und Lehre gälte dann überdies, daß bestimmte Argumentationen und Ergebnisse im öffentlich geförderten Wissenschaftsbetrieb von vornherein negativ sanktioniert wären.

3. Für die wissenschaftliche Forschung und Lehre ist ein weiteres wichtig: Theorten der Verfassungswirklichkeit der Bundesrepublik Deutschland können nicht von vornherein aut ein positives oder neutrales Urteil zum Grundgesetz festgelegt werden. Das gilt auch für unabänderliche Grundgesetzartikel und für 
Prinzıpien, die $1 \mathrm{~m}$ Urteil des Bundesverfassungsgerichts vom 23.10.1952 als Verpflichtungsbasis für die Angehörıgen des Offentlichen Dienstes genannt werden; soweıt es sıch dabei nıcht um Formeln für einen allgemeinen moralischen Rahmen handelt, den auch eine rational verstandene Wissenschaft nicht verlassen kann. Insbesonders muß es möglich sein, in vergleichenden historischen und empirischen Analysen auf kritikbedürftige Konsequenzen unabänderlicher Grundgesetzregelungen im Zusammenwirken etwa mit Normen der Wirtschaftsordnung hinzuweısen. Würden derartıge Theorien nıcht in den Bereich des Freiheıtsgebotes von Art. 5, Abs. 3 Grundgesetz fallen, so würde sich die Verfassung gegenüber der Wissenschaft in diesem Punkte die Position des Kardinals Bellarmin gegenüber Galilei anmaßen. Dies kann nıcht ım Sinne der wissenschaftsbezogenen Freiheitsgarantie des Grundgesetzes liegen. Auch die prinzipiellen Behauptungen und Meinungen, die den Konstruktionen des Grundgesetzes zugrundeliegen, sind demnach für eine wissenschaftliche Erörterung, die nicht bestätigenden Charakter hat, nicht tabu. Selbst soweit wissenschaftliche Theorien solche Grundgesetzkritik enthalten, kann damit die Einstellung ihrer Autoren in den Offentlichen Dienst wissenschaftlicher Forschung und Lehre nur nach denselben Maßstäben erfolgen, die für Wissenschaftler, deren Arbeiten einen anderen Gegenstand haben, angelegt werden. Ein Fall, den die Freiheitsgarantie von Grundgesetzartikel s, Abs. 3 nicht mehr deckt, liegt erst dort vor, wo aus einer theoretischen Grundgesetzkritik die nicht-theoretısche Konsequenz eines verfassungs- und gesetzwidrigen Handelns gezogen wird.

4. Für wissenschaftliche Institutionen wie für die demokratische Grundordnung gilt gleichermaßen, daß jede Einschränkung der Freiheiten des Art. 5 ihren Lebensnerv berührt. $\mathrm{Da}$ die wissenschaftlichen Institutionen in der Bundesrepublik Instıtutionen des Offentlichen Dienstes sind, widerspricht jede Ablehnung von Bewerbern für den Offentlichen Dienst im Wissenschaftsbereıch aufgrund von nicht gerichtsverwertbaren politıschen *Erkenntnissen Art. I2, Abs. I Grundgesetz und stellt überdies eine Einschränkung der durch die Verfassung garantierten Freiheit von Forschung und Lehre dar. Eine Auslese von Bewerbern nach Kriterien politischer Zuverlässigkeit mag einer politisch gelenkten staatlichen Bürokratıe als geeıgnetes Mittel erscheinen, die Freihe1t der Wissenschaft und die demoksratische Grundordnung zu schützen; in Wirklichkeit ist es eın sicheres Mittel, beide zu zerstören.

5. Eine Beurteilungspraxis im Zusammenhang mit der Einstellung in den Offentlichen Dienst, die sich den oben in Erinnerung gerufenen Grundsätzen verpflichtet hat, wird als nichttotalitäre überdies den Betroffenen nicht ihre vergangenen Erklärungen und Auffassungen vorhalten können. Wenn sie nicht den total irrtumsfreien Menschen fordern will, muß sie auch bes politischen und verfassungshermeneutischen Aussagen zum Grundgesetz das gegenwärtig artikulierte Verständnıs der Betroffenen zugrundelegen, wenn diese nicht glaubhaft widerlegt werden kann. Es verstößt insbesondere gegen die Basısnormen des Rechtsstaates, hier die Beweislast dem Betroffenen aufzubürden.

Es sollte enne bittere historische Erfahrung gerade in Deutschland gelehrt haben, daß die Bedrohung eıner verfassungsmäßigen demokratischen Grundordnung auch von staatlichen Bürokratien ausgehen kann. Es sei daran erınnert, daß die Verpflichtung zur Verfassungstreue für öffentliche Bedienstete auch die Pflicht einschließt, sich gegen Verletzungen der Verfassung von seiten der staatlichen Bürokratie zur Wehr zu setzen. Dies gilt insbesondere dann, wenn im Namen eines Schutzes der demokratischen Grundrechte in der auf die 
sogenannten Radikalenerlasse gegründeten Ausforschungs-, Beurteilungs- und Ablehnungspraxis. Die unmittelbare Folge ist ein erheblicher Einschüchterungseffekt in den Berufsfeldern des Offentlichen Dienstes. Diese für eine an ennem aufgeklärten Verfassungsverständnis orıentierte Berufspraxis verheerende Folge kann nicht die Absıcht der Landesregierungen seın. In der durch die Verfassung gebotenen Wahrnehmung ihres Amtes fordern daher die Unterzeichner insbesondere die Landesregierung Baden-Württembergs auf, die zur Zeit von ihr geübte Uberprüfungs- und Einstellungspraxis $1 \mathrm{~m}$ Sinne dieser Erklärung zu revidieren.

\section{Zeugnisverweigerungsrecht nur für die Sozialarbeiter der Reichen?}

Durch Beschluß vom I 9. Juli 1972 hatte der 2. Senat des Bundesverfassungsgerichts die Verweigerung eines strafprozessualen Zeugnisverwergerungsrechts der Sozialarbeiter für verfassungsgemäß erklärt ${ }^{1}$. Dieser Beschluß stıeß nicht nur auf Kritik bei den Sozıalarbeitern². Auch Teile der Öffentlichkeit nahmen ihn nicht widerspruchslos hın. So erklärte beispielsweıse der Unterbezirk Frankfurt der SPD: "Als Klassenjustiz entpuppt sich dieser Beschluß, wenn er daneben das Zeugnısverweigerungsrecht von Patentanwälten, Wirtschaftsprüfern, vereidigten Buchprüfern, Steuerberatern und Steuerbevollmächtigten ... rechtfertigt... Die jetzige Regelung stellt eine eindeutige Benachteiligung derjenigen Bevölkerungsgruppen dar, die sıch aufgrund ihrer sozialen Lage und Herkunft nicht an eine mit finanziellem Aufwand verbundene Instanz (Psychotherapeut, Rechtsanwalt usw.) wenden können. "3 Aufgrund dieser Kritik nahm der SPD-Parteitag in Hannover 1973 einen Antrag des Unterbezirks Frankfurt an, der die SPD-Mitglieder in Bundesregierung und Bundestag autfordert, »die Diskriminierung von Nicht-Privilegierten in $\$ 53 \mathrm{StPO}$ aufzuheben und noch in dieser Legislaturperiode das Zeugnisverweigerungsrecht auf alle Personen auszudehnen, deren psychotherapeutische oder sonst beratende Tätigkeıt ein besonderes menschliches Vertrauensverhältnis voraussetzt oder in aller Regel begründet. Dazu zählen z. B. Psychotherapeuten, Psychologen, Sozialpädagogen, Sozialarbeiter, Bewährungshelfer, amtliche Berater in Familienangelegenheiten usw. " ${ }^{+}$

Nicht zuletzt aufgrund dieses Parteitagsbeschlusses sah sich die Bundesregierung zu einer Regelung des Zeugnisverweigerungsrechts für die genannten Berufsgruppen gezwungen. Am 6.9. I 974 legte sie im Rahmen des „Entwurfs eines zweiten Gesetzes zur Refom des Strafverfahrensrechts (2.StVRG) einen neuen $₫ 53$ I Nr. 3a StPO vor. Danach sollen ein Zeugnisverweigerungsrecht erhalten "staatlich anerkannte Sozıalarbeiter, staatlich anerkannte Sozialpäd-

\footnotetext{
1 BVerfGE 33, S. 367 if.

2 Vgl. z. B. die Stellungnahmen in Neue Praxıs, Krıtısche Zeıtschrift für Sozıalarbeıt und Sozıalpädagogik $2 / 73$, S. 89 ff., $3 / 73$, S. 257 ff., $1 / 74$, S. 69 ff.; Aussagezwang für Sozıalarbeiter, in: Rote Robe 6/72, S. 272 f., Rechtsprechung BVertG: Kenn Aussageverwe1gerungsrecht für Sozılarbeiter, in: Sozıalpädagogische Korrespondenz 26/72, S. Sff.; betrifft Zeugnisverweıgerungsrecht, in: Sozıalpädagrogische Korrespondenz $31,32 / 73,5.33 \mathrm{ff}$.

3 Zitiert nach Neue Praxis 3/73, S. 263.

+ Antrag 128 Abs. 1, zitiert nach Neue Praxis a. a. O., (Fn. 3).
} 